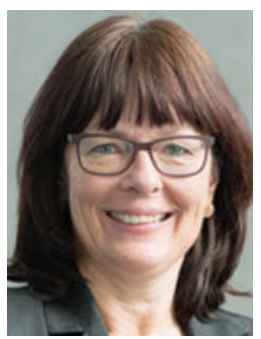

Prof. Dr. rer. medic. Ute Lange

Hebamme und Professorin für Hebammenwissenschaft an der Hochschule für Gesundheit in Bochum

\section{Frauen mit Handicap kompetent begleiten}

\section{Wie Frauen im Rollstuhl Schwangerschaft, Geburt und Mutterrolle erleben}

Die 2018 veröffentlichte S2k-Leitlinie zur geburtshilflichen Betreuung bei Querschnittlähmung unterstützt Ärzte und Ärztinnen sowie Hebammen bei einer angepassten und kompetenten Versorgung betroffener Frauen. Im aktuellen Heft stellen wir Ihnen die Leitlinie vor und fokussieren auf Aspekte, die für die Hebammenarbeit besonders relevant sind. In einem weiteren Artikel werden grundlegende medizinische Informationen zur Thematik der Querschnittlähmung ergänzt.

Einen besonderen Schwerpunkt dieses Themenhefts bilden die Sichtweisen und Erlebnisse der Frauen mit Querschnittlähmung: Wir freuen uns, dass uns mehrere Mütter von ihren Erfahrungen in Schwangerschaft, Geburt und Wochenbett berichtet haben und dem Heft damit eine wichtige Perspektive hinzufügen. Hilfreiche Adressen zur Selbsthilfe und Hinweise auf Hilfsmittel zur Alltagsunterstützung im Leben mit dem Säugling ergänzen dieses Themenheft.

Wir hoffen, dass die Artikel eine hilfreiche Unterstützung bei der Begleitung von Frauen mit Querschnittlähmung bieten und den Blick auf die Mütter und Familien erweitern, die trotz besonderer Herausforderungen ihr Recht auf ein und den Wunsch nach einem Leben mit Kind(ern) umsetzen und leben.

Viel Spaß beim Lesen wünscht Ihnen

Ute Lange

Herausgeberin DIE HEBAMME

\section{Herausgeber-Team}

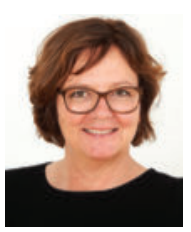

Heidi Bernard

Hebamme, Lehrerin für Pflege und Gesundheit M.A., Leitung Elternschule Neue Kölner, Köln

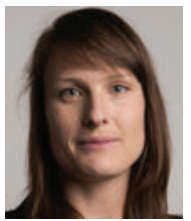

Daniela Garten

Leitende Hebamme, B.Sc. Midwifery, Städtisches Klinikum Dresden

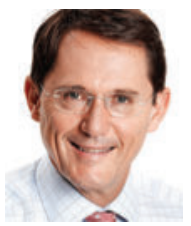

Prof. Dr. med. Franz Kaine

Chefarzt der Abteilung für Geburtshilfe und Pränatalmedizin, Klinik Hallerwiese, Nürnberg, Präsident der Deutschen Gesellschaft für Perinatale Medizin

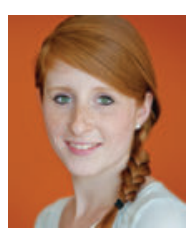

Anne-Katrin Klotzsch

Hebamme B.Sc., qualifizierte Praxisanleiterin, Kreißsaal St. Joseph Krankenhaus, Berlin-Tempelhof

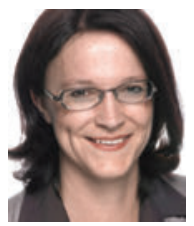

Dr. rer. medic. Astrid Krahl

Dipl. Pflegewirtin (FH), Hebamme, Studiengangleitung M.Sc. Hebamme, ZHAW Gesundheit, Institut für Hebammen, Winterthur

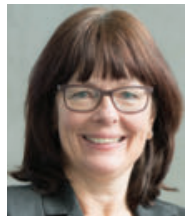

Prof. Dr. rer. medic. Ute Lange

Hebamme und Professorin für Hebammenwissenschaft an der Hochschule für Gesundheit in Bochum 